\title{
Fabrication of a Gradient Nano-/Micro-structured Surface Layer on an Al-Si Casting Alloy by Means of Ultrasonic-Electropulsing Coupling Rolling Process
}

\author{
Xiao-Pei $\mathrm{Li}^{1,2,3} \cdot$ Song-Zhu Kure-Chu ${ }^{3} \cdot$ Toru Ogasawara $^{3} \cdot$ Hitoshi Yashiro $^{3} \cdot$ Hai-Bo Wang ${ }^{1} \cdot \mathrm{Zi}^{-Z h e n} \mathrm{Xu}^{1} \cdot$ \\ Xiao-Hui $\mathrm{Li}^{1} \cdot$ Guo-Lin Song ${ }^{1} \cdot$ Guo-Yi Tang ${ }^{1,2}$
}

Received: 3 January 2018/Revised: 7 February 2018/Published online: 26 April 2018

(C) The Chinese Society for Metals and Springer-Verlag GmbH Germany, part of Springer Nature 2018

\begin{abstract}
By using a novel surface modification technique named ultrasonic-electropulsing coupling rolling (UECR) process on an Al-Si casting alloy rod, the surface of material was smoothened significantly. Meanwhile, a strengthened layer with a gradient change in hardness was obtained in the outer surface, corresponding to a homogeneous gradient nano-/microstructure. The thickness of nanometer-thick laminated structures was at least $40 \mu \mathrm{m}$, which was much thicker than conventional ultrasonic rolling process. During UECR, the formation of the well-defined nanocrystalline structure was attributed to the high strain rate and simultaneous annealing process realized by ultrasonic impact and electropulsing treatment.
\end{abstract}

Keywords Nanocrystalline materials $\cdot$ Indentation and hardness $\cdot \mathrm{Al}-\mathrm{Si}$ alloy $\cdot$ Microstructure

\section{Introduction}

Nanocrystalline (NC) materials have been found to possess attractive properties over their coarse-grained polycrystalline counterparts [1]. For decades, the various techniques have been employed to fabricate bulk NC materials, including electrodeposition [2], inert gas condensation [1], severe plastic deformation (SPD) processing [3-5]. However, most of the current techniques for bulk NC materials fabrication are not suitable for industrial scale due to limitations in terms of products size and cost. In comparison with bulk NC materials fabrication, the surface

Available online at http://link.springer.com/journal/40195

Song-Zhu Kure-Chu

chusongz@iwate-u.ac.jp

$\triangle$ Guo-Yi Tang

tanggy@mail.tsinghua.edu.cn

1 Advanced Materials Institute, Graduate School at Shenzhen, Tsinghua University, Shenzhen 518055, China

2 School of Materials Science and Engineering, Tsinghua University, Beijing 10084, China

3 Department of Chemistry and Bioengineering, Iwate University, Iwate 020-8550, Japan nanocrystallization (SNC) could be achieved by simple processes with less cost of energy and greatly enhance the global performance of materials at the same time $[6,7]$ and thereby is potentially very useful in industrial applications. Several techniques have been developed based on this principle: surface mechanical attrition treatment [8], friction sliding [9], surface mechanical grinding treatment $[3,10]$, ultrasonic surface rolling process [11], ultrasonic impact treatment $[12,13]$, etc. Chen et al. [13] compared the respective effects of mechanical and ultrasonic impact on the microstructures of the welded joint of $2195 \mathrm{Al}-\mathrm{Li}$ alloy and found that the mechanical impact could not refine the grains, but the ultrasonic impact could refine the grains, indicating the effectiveness of ultrasonic in grain refinement. Unfortunately, these processes suffer from either low processing efficiency or limited nanostructured layer thickness or structural inhomogeneity in the surface layer [3]. And the remarkable hardening, as well as large amounts of dislocations, in the surface-strengthened layer generated during severe cold working acts as an extreme obstruction to the further deformation and strengthening inside the materials. All of the above have hindered the widespread application of SNC technologies.

Recently, as a novel surface modification technique based on SPD processing, the ultrasonic-electropulsing 
coupling rolling (UECR) process has been noticed to be an effective and efficient way to enhance the surface properties of metallic materials, including roughness, hardness and wear resistance $[14,15]$. Actually, the idea of joint use of ultrasonic treatment and electropulsing has been proposed by Troitskii [16], which was proved to be effective in pressure shaping. With the addition of electropulsing to conventional ultrasonic surface rolling process, hardness and even the depth of strengthened layer are enhanced comparing with the conventional ultrasonic surface rolling process [17], which is attributed to the promoted dislocation mobility and subgrain boundary migration by electropulsing [18, 19]. This promotion mechanism was also reported by $\mathrm{Li}$ et al. [20] that the dislocation movement was promoted by pulse current, resulting in the superplastic deformation of AZ31 magnesium alloy. Although so many advantages of UECR have been reported, the microstructure characterization of the processed sample, especially the topmost surface, is still insufficient, which directly hinders the understanding of grain refining mechanism, as well as process optimization of UECR. In the present study, UECR was utilized to modify an Al-12Si casting alloy, which has received considerable attention in automotive and aerospace industries, and especially some wearrelated applications such as pistons and pivots in internal combustion engines [21, 22]. Consequently, the enhancements of the surface finish qualities, i.e., hardness and roughness, as well as mechanical properties, are important to its application performance. For comparison, conventional ultrasonic rolling (UR) process, i.e., without using electropulsing, was also carried out.

\section{Experimental}

The Al-12Si casting alloy rods (Al-12\% $\mathrm{Si}-3.5 \% \mathrm{Cu}-$ $2 \% \mathrm{Ni}$ ) were received as starting material. Turning process was employed to the rods before rolling to obtain a uniform sample dimension of $\Phi 12.5 \mathrm{~mm} \times 80 \mathrm{~mm}$ and uniform surface morphology characteristics. The UECR and UR experiments were conducted on a self-built platform based on a conventional lathe. The schematic view of the process can be found in Ref. [15]. The basic parameters related to UECR were as follows: The rotate speed was $200 \mathrm{rpm}$, ultrasonic vibration frequency was $30 \mathrm{kHz}$, and feeding rate was $0.1 \mathrm{~mm} / \mathrm{rev}$, i.e., the ultrasonic apparatus advances $0.1 \mathrm{~mm}$ per revolution. For UECR, the root-mean-square current density and peak current density and duration of electropulsing were $\sim 10^{2}, \sim 10^{3} \mathrm{~A} / \mathrm{cm}^{2}$ and $60 \mu \mathrm{s}$, respectively. The surface temperature of the $\mathrm{Al}$ alloy rod during UECR, monitored by using a $K$-type surface thermocouple, was $\sim 120{ }^{\circ} \mathrm{C}$, which was primarily caused by the Joule heating effect of electropulsing, while for UR, the surface temperature during processing was $\sim 40{ }^{\circ} \mathrm{C}$. This slightly increased temperature was attributed to high-speed friction between the sample and the ultrasonic apparatus.

The surface profiles of sample before and after UECR were measured by a surface roughness meter (Taylor Hobson, Form Talysurf PGI 2000S). The cross-sectional Vickers microhardness within the surface layer was measured by a microhardness tester (Mitutoyo) employing a 30 -g load for a dwell time of $30 \mathrm{~s}$. Nanoindentation hardness was tested by Nano Indenter G200. Electron backscattered diffraction (EBSD) analyses were carried out using a field-emission scanning electron microscope (FESEM, JEOL JSM-7001F). A JEM-2100 transmission electron microscope (TEM) was utilized to characterize the nanocrystallites in the topmost surface. Samples for TEM were prepared by focused ion beam (FIB).

\section{Results and Discussion}

Figure 1 shows the surface morphology images and surface profiles of the $\mathrm{Al}$ alloy rod before and after UECR. As can be seen, the rod exhibits a mirror-like bright surface after UECR, with an almost flat surface profile comparing with the turning surface. Meanwhile, average roughness value (Ra) decreases from 1.8641 to $0.0778 \mu \mathrm{m}$ after modification. A rather smooth rolling surface generates from the rugged turning surface, as displayed in the SEM images, indicating the outstanding surface smoothening effect of UECR. Besides, the surface profile in Fig. 1c, acquired from the region between the turning and rolling surface, shows a rather smooth transition without any obvious steps, which is very significant in industrial application.

The cross-sectional microhardness distributions before and after rolling process are presented in Fig. 2a. As anticipated, no obvious influence on the microhardness of Al alloy rod has been caused by the initial turning, with its value falling in the range of $100 \pm 10 \mathrm{Hv}$. In contrast, after UECR, an obvious strengthened layer of more than $200 \mu \mathrm{m}$ thick is obtained. In the top $\sim 40-\mu \mathrm{m}$-thick layer, the hardness increases to $\sim 200 \mathrm{Hv}$, which is nearly twice as much as it for the original state. As the depth increases, hardness decreases gradually and finally returns to $\sim 100$ $\mathrm{Hv}$ in $\sim 220 \mu \mathrm{m}$ depth, whereas for the conventional UR, the strengthening effect seems much weaker than UECR in terms of both hardness and impact depth. Although the hardness of the top $\sim 40$ - $\mu \mathrm{m}$-thick layer receives a considerable improvement to $\sim 160 \mathrm{Hv}$, it is still much smaller than UECR-processed sample. And the hardness returns to the original state in $200 \mu \mathrm{m}$ depth, indicating the thickness of strengthened layer is less than $200 \mu \mathrm{m}$. The nanoindentation hardness shows similar results. For UECR sample, hardness is largely increased in 40 and $80 \mu \mathrm{m}$ 


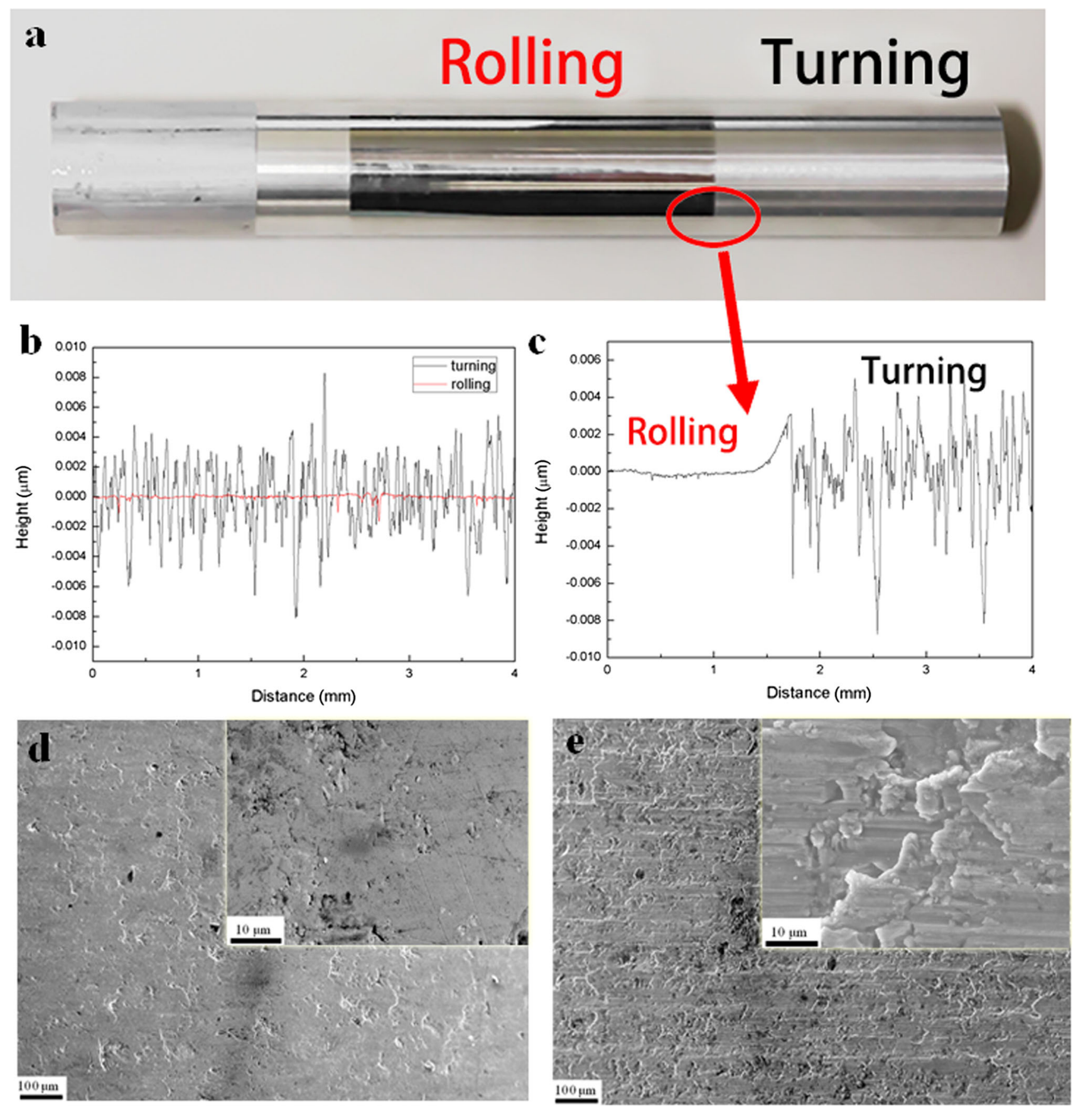

Fig. 1 a Digital photograph of the processed Al alloy rod, b surface profiles of the rolling surface and the turning surface, $\mathbf{c}$ surface profile measured in the transition area between the rolling and turning surface, $\mathbf{d}$, e respective SEM images of rolling surface and turning surface. Turning and rolling refer to the turning surface before UECR and the rolling surface after UECR, respectively

depth. But for UR sample, evident enhancement of hardness is only found in $40 \mu \mathrm{m}$ depth. In $80 \mu \mathrm{m}$ depth, increase in hardness is so slight that its curve almost overlaps with the $200 \mu \mathrm{m}$ depth one.

Interestingly, the microstructure evolution exhibits some similarities to the microhardness gradient. Before rolling process, the microstructure consists of coarse grains with grain sizes of more than $100 \mu \mathrm{m}$, which is the typical microstructure for casting alloy. The small misorientation close to the treated surface, indicated by color variation, is induced by machining stress during turning. And after UECR, the typical casting microstructure, noticeably, is replaced by a gradient structure with fine grains (Fig. 2d). For the top $\sim 40-\mu \mathrm{m}$-thick layer, the microstructure is characterized by typical laminar fine grain structure, which may attribute to the severe shear effect close to the treated 

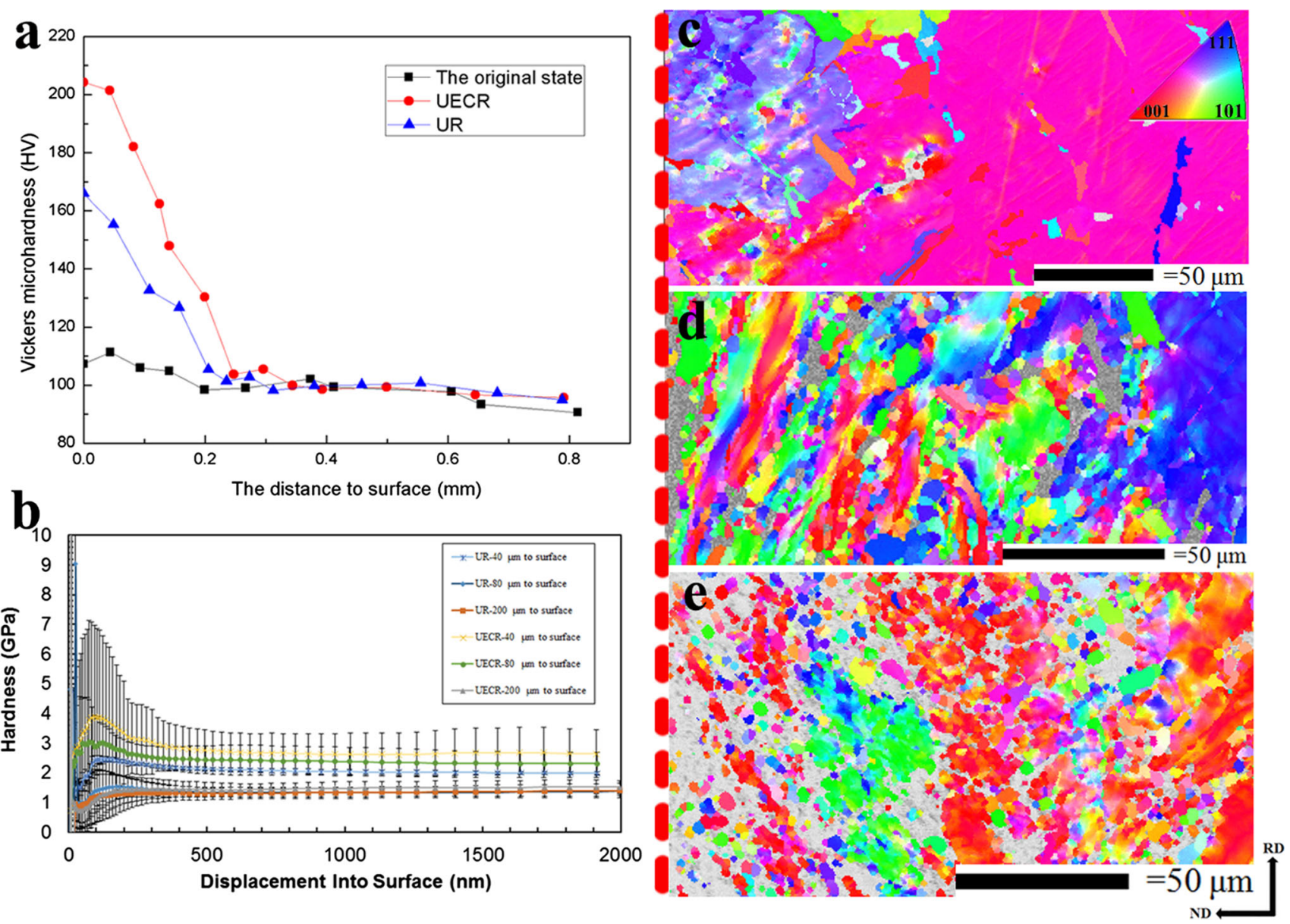

Fig. 2 a Cross-sectional microhardness distribution of the Al alloy rods, $\mathbf{b}$ nanoindentation hardness for UECR and UR sample in different depths to surface, $\mathbf{c}-\mathbf{e}$ inverse pole figure (IPF) maps calculated from EBSD data of the Al alloy rod in the original state and after UECR and UR, respectively. The treated surface is outlined by a red dashed line. The RD refers to the rolling direction, and the ND refers to the normal direction

surface and the impact effect of ultrasonic treatment. With the depth increasing, both the shear and impact effect weaken; thereby, the laminar structure becomes less evident and grain size also increases gradually. Finally, after $120 \mu \mathrm{m}$ depth, grain sizes become comparable to the casting microstructure; however, there exists profuse substructure inside these coarse grains. It is obvious that, after UECR, the microstructure gradient is well consistent with the microhardness gradient in Fig. 2a. For the top $\sim 40-$ $\mu \mathrm{m}$-thick layer, microstructure is composed of fine laminar grains, and the microhardness is thereby increased remarkably according to the Hall-Petch relation. Afterward, grain size increases gradually with depth increasing, accompanied by continuous decrease in hardness. Figure $2 \mathrm{e}$ also gives the microstructure of conventional URprocessed sample. It can be found that the grains exhibit an equiaxed feature throughout the strengthened layer. Therefore, grain morphology of the UR sample is quite different from the UECR sample, which contains a gradient structure from laminar fine grain to equiaxed fine grain as the depth increases.

From the TEM observations in Fig. 3, both nanometerthick laminated (NL) structures and equiaxed ultrafinegrained (UFG) structures are obtained from the Al alloy rod after UECR, which is primary depended on the depth to the treated surface. It is found that microstructure of the top layer up to $40 \mu \mathrm{m}$ depth is characterized by NL structures (Fig. 3a, b). And the corresponding selected area electron diffraction (SAED) pattern indicates that the NL grains are randomly oriented. The transverse axis sizes of most NL grains observed in Fig. 3a, b are less than $100 \mathrm{~nm}$. And in $80 \mu \mathrm{m}$ depth, the NL structures are replaced by almost equiaxed UFG structures with sizes of up to a few hundred nanometers. That is to say, a nanostructured layer with a thickness of at least $40 \mu \mathrm{m}$ is achieved on the Al alloy rod by using UECR, which is much thicker than that obtained by means of ultrasonic impact treatment $(\sim 10 \mu \mathrm{m})$ [12], wire brushing $(\sim 15 \mu \mathrm{m})[6]$ and ultrasonic shot peening $(\sim 20 \mu \mathrm{m})$ [23], indicating that grain refinement is more 

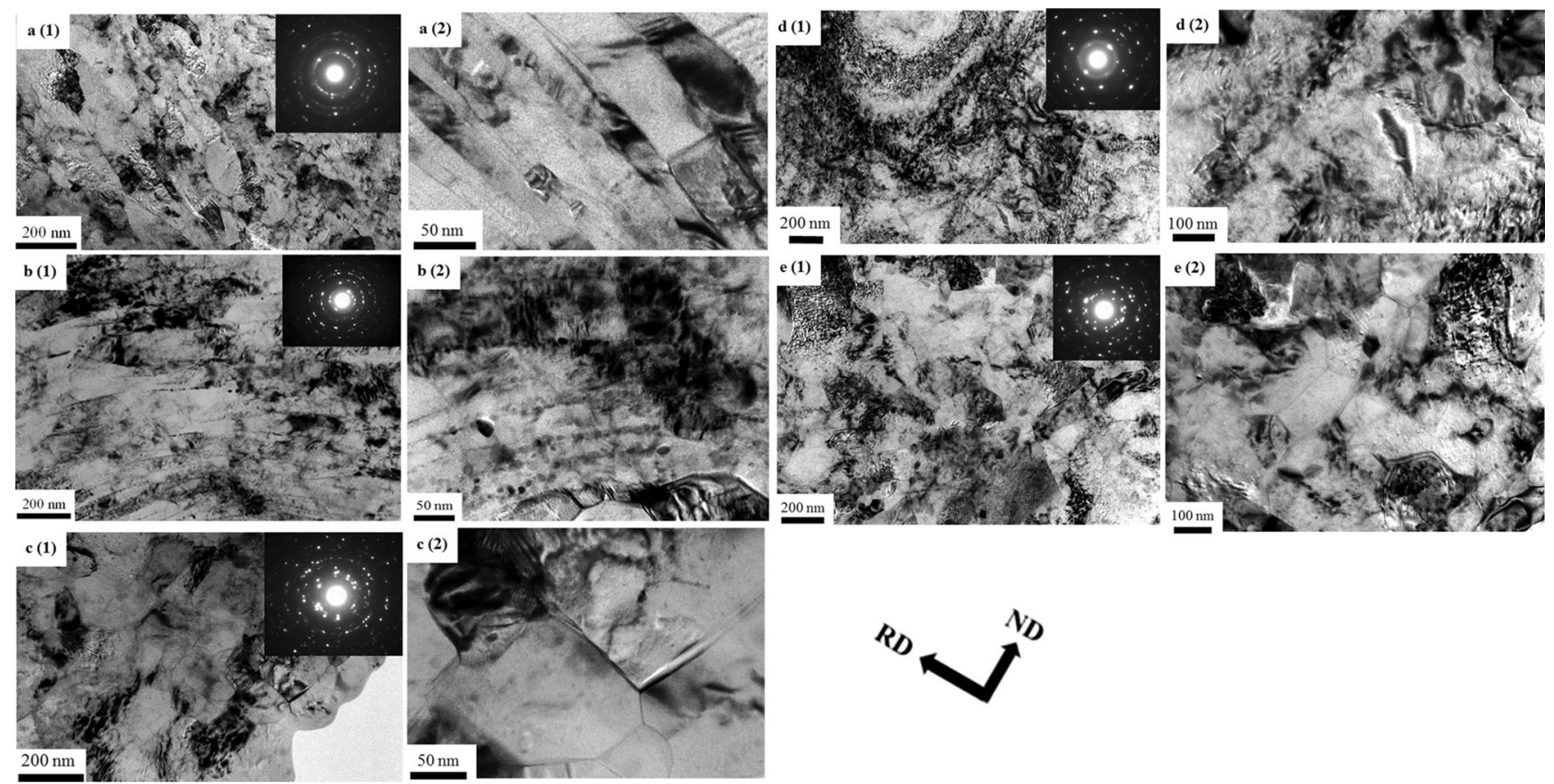

Fig. 3 TEM observations of the Al alloy rod after UECR and UR: a-c for the UECR sample, a NL structures obtained from $10 \mu \mathrm{m}$ deep to the surface, b NL structures obtained from $40 \mu \mathrm{m}$ deep to the surface, $\mathbf{c}$ UFG structures obtained from $80 \mu \mathrm{m}$ deep to the surface; d, e for the UR sample, $\mathbf{d}$ heavily deformed microstructure obtained from $10 \mu \mathrm{m}$ deep to the surface, e UFG structures obtained from $40 \mu \mathrm{m}$ deep to the surface. $\mathbf{a} 2, \mathbf{b 2}, \mathbf{c 2}, \mathbf{d 2}, \mathbf{e} 2$ are the respective magnified views of $\mathbf{a 1}, \mathbf{b 1}, \mathbf{c 1}, \mathbf{d 1}, \mathbf{e 1}$

effective in the present UECR. But for UR-processed sample, the observations are quite different. Figure 3(d) shows no typical NL structures as UECR-processed sample. Moreover, we can even hardly identify any substructure boundaries due to the high density of dislocations. It seems that the obvious grain refinement is not achieved in the top layer. The corresponding SAED pattern shows an obscure but discernable amorphous ring, indicating some of crystal lattices are so heavily distorted that the lattices lose the feature of crystal. And in $40 \mu \mathrm{m}$ depth, the microstructure is characterized by equiaxed UFG structures, just as the structure of UECR-processed sample in $80 \mu \mathrm{m}$ depth. The grain sizes are also up to a few hundred nanometers. That is to say, the thickness of obtained nanostructured layer by conventional UR will be no more than $40 \mu \mathrm{m}$.

Generally, the fabrication of nanocrystalline metallic materials by conventional SPD methods is conducted at ambient temperature for the reason that the nanocrystalline structure tends to coarsen at elevated temperature due to its high interfacial energy. However, the heavily cold working is likely to induce numerous defects into the material (e.g., point defects, stacking fault and microcrack), which may significantly influence the mechanical behavior of the products $[8,24,25]$. Consequently, some researchers suggested that moderate annealing without causing noticeable grain growth, i.e., low temperature and short annealing time, was essential to enhance the mechanical behavior of SPDed NC materials [24, 26].

Electropulsing treatment (EPT), as a substitute for conventional heat treatment, has been noticed to result in fast evolution of the microstructure of treated material due to its positive effects on dislocation mobility and subgrain boundary migration $[18,27]$. In the present study, $\mathrm{Al}$ alloy rod was subjected to ultrasonic rolling and electropulsing treatment simultaneously. It seems that the drastic strain induced by ultrasonic impact has been mostly accommodated, as can be seen in Fig. 4a. After UECR, coarse grains are split into fine grains layer by layer, and subgrain boundaries continue to divide these grains into laminated structure. The well-ordered subgrain boundaries are quite different from those discovered in conventional SPDed materials, which usually contain a high density of crystallographically disordered grain boundaries (GBs) [5]. Besides, from the TEM images in Fig. 4b, c, dislocations are piled up and well-defined NL structures are formed, whereas for UR sample (Fig. 4d-f), the well-defined NL structures are not found. The rearrangement of dislocations and formation of low-angle subgrain boundaries, known as "recovery," thereby, can only be attributed to electropulsing. Song et al. [19] found pulse current could decrease the dislocation density and pileups and improve the dislocation movement, which was primarily attributed to the dominant role of Joule heating effect of pulse current. However, the athermal effect of electropulsing has 

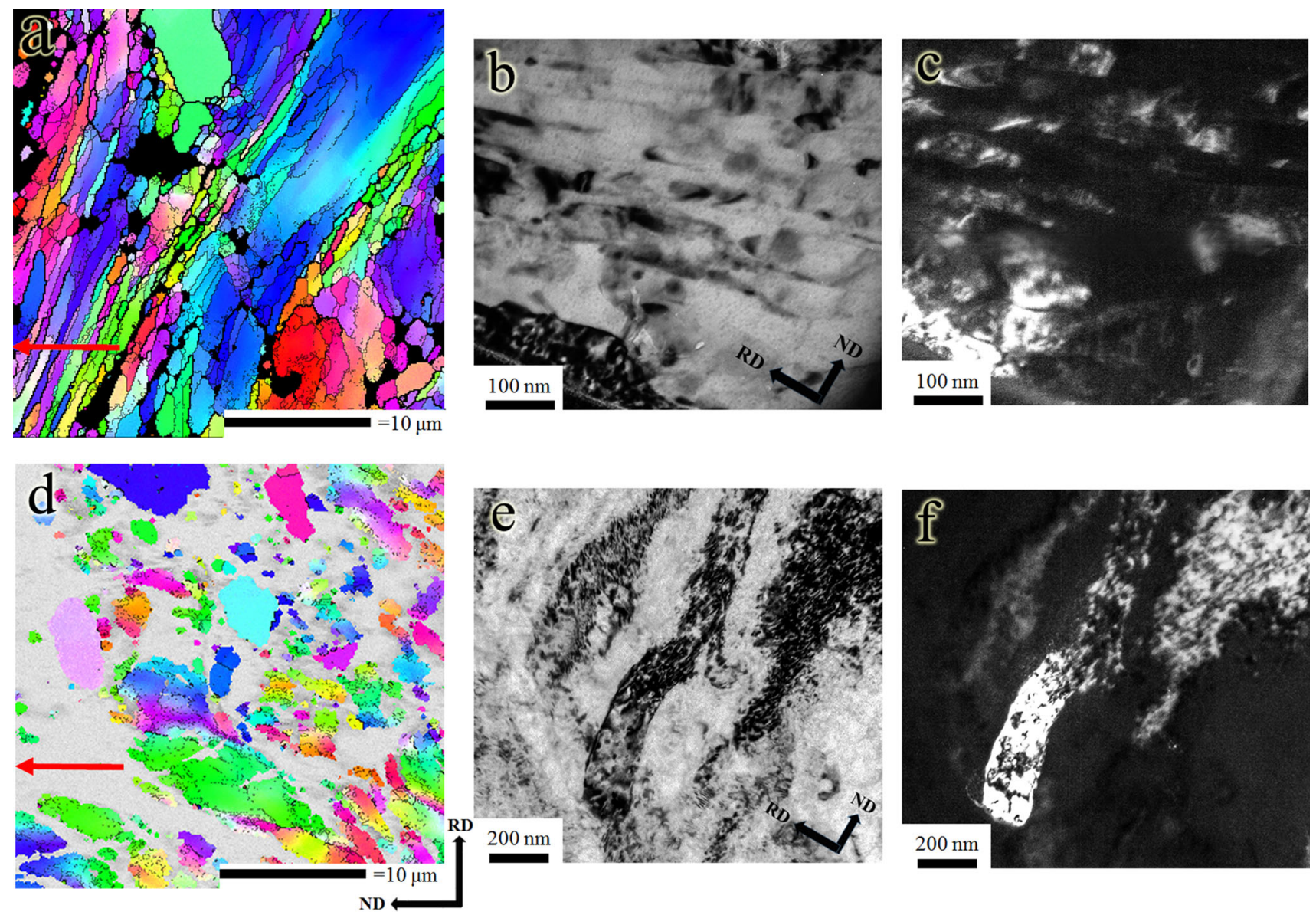

Fig. 4 a, d Magnified IPF map of the top 30- $\mu$ m-thick layer, in which the thin black lines indicate boundaries of misorientation between $2^{\circ}$ and $15^{\circ}$, and the thick black lines indicate boundaries above $15^{\circ} ; \mathbf{b}, \mathbf{e}$ bright-field image, $\mathbf{c}, \mathbf{f}$ corresponding dark-field image. The red arrows in $\mathbf{a}$, $\mathbf{d}$ indicate the treated surface. $\mathbf{a}-\mathbf{c}$ are for the UECR sample, and $\mathbf{d}-\mathbf{f}$ are for the UR sample

also been reported by many researchers [28-30]. In addition to the commonly accepted electron wind force of electropulsing, $\mathrm{Li}$ et al. [30] recently reported the effect of selective heating at the dislocation cores, which would also result in an increased dislocation mobility. Consequently, both the thermal effect and athermal effect of electropulsing contribute to the piled-up dislocations and the formation of well-defined NL structures during UECR. Also, it is believed that the well-defined NL structures are responsible for the increased hardness of UECR sample comparing to UR sample. Annealing-induced hardening phenomena have already been observed in other nanostructured samples [26, 31, 32]. Tong et al. [24] found that the moderate annealing or recovery treatment not only increased the strength of the NC $\alpha$-iron by reducing dislocation emission sources but also improved its ductility through strengthening the GB's resistance to intergranular cracking.

Another effect of electropulsing mentioned above is increasing the thickness of strengthened layer, especially the nanostructured layer. With regard to the conventional
UR-processed sample, as the IPF map and TEM observations show, its top surface has suffered from severe cold working and contains large amounts of dislocations. The dislocation pileup and tangling result in remarkable hardening in the topmost surface-strengthened layer and act as an extreme obstruct for the further plastic deformation and strengthening inside of the alloy [14]. As a consequence, the thickness of both strengthened layer and nanostructured layer is rather limited, whereas for UECR, it has already been reported that electropulsing is able to untie the dislocation tangling and facilitate the accumulation of dislocations in the vicinity of GBs and form substructures $[33,34]$. In this way, plastic deformation and strengthening effect toward the interior of material will be much easier. Moreover, recently, Qin [35] found that electropulsing can be used to surpass the microstructure breakup limit and, therefore, realize microstructure refinement for the reason that the breakup causes the electric current free energy to reduce in some cases, which compensates the increment of interfacial free energy during breaking up and enables the processing to achieve finer microstructure. We believe that 
this effect of electropulsing also contributes to microstructure refinement during UECR.

\section{Conclusion}

UECR is an effective and efficient method to enhance the surface finishing qualities on bulk metallic materials basing on surface nanocrystallization. On an Al-Si casting alloy rod processed by UECR, the thickness of obtained NL structures is at least $40 \mu \mathrm{m}$, which is much thicker than that for conventional UR. The introduction of electropulsing not only enhances the hardness of strengthened layer through the annealing-induced hardening effect, but also increases the thickness of strengthened layer.

Acknowledgements The work is financially supported by the Shenzhen Nanshan District Governmental Foundation (Grant No. KC2015ZDYF0021A), the Shenzhen Industry Development Foundation (Grant No. HYCYGJ20140512010015) and the Shenzhen Development and Reform Commission Foundation (Grant No. 2015-1033). The authors appreciate Kuniaki Sasaki's help with EBSD and TEM samples preparation.

\section{References}

[1] H. Gleiter, Prog. Mater. Sci. 33, 223-315 (1989)

[2] N. Yang, T. Headley, J. Kelly, J. Hruby, Scr. Mater. 8, 51 (2004)

[3] W. Li, N. Tao, K. Lu, Scr. Mater. 5, 59 (2008)

[4] J. Villegas, L. Shaw, Acta Mater. 19, 57 (2009)

[5] S. Zheng, J.S. Carpenter, R.J. McCabe, I.J. Beyerlein, N.A. Mara, Sci. Rep. 4, 4226 (2014)

[6] M. Sato, N. Tsuji, Y. Minamino, Y. Koizumi, Mater. Sci. Forum 426, 2753-2758 (2003)

[7] G. Liu, J. Lu, K. Lu, Mater. Sci. Eng. A 1, 286 (2000)

[8] H.W. Chang, P. Kelly, Y.N. Shi, M.X. Zhang, Surf. Coat. Technol. 19, 206 (2012)

[9] D. Hughes, N. Hansen, Phys. Rev. Lett. 13, 87 (2001)
[10] J.M. Xu, Y. Liu, B. Jin, J.X. Li, S.M. Zhai, X.J. Yang, J. Lu, Acta Metall. Sin. (Engl. Lett.) 9, 28 (2015)

[11] W. Ting, W. Dongpo, L. Gang, G. Baoming, S. Ningxia, Appl. Surf. Sci. 5, 255 (2008)

[12] X. An, C. Rodopoulos, E. Statnikov, V. Vitazev, O. Korolkov, J. Mater. Eng. Perform. 3, 15 (2006)

[13] Q.H. Chen, S.B. Lin, C.L. Yang, C.L. Fan, Acta Metall. Sin. (Engl. Lett.) 4, 29 (2016)

[14] H. Wang, G. Song, G. Tang, Surf. Coat. Technol. 25, 282 (2015)

[15] H. Wang, G. Song, G. Tang, J. Alloys Compd. 5, 681 (2016)

[16] O. Troitskii, Mater. Sci. Eng. 1-2, 75 (1985)

[17] T. Liu, X. Li, G. Tang, G. Song, J. Mater. Res. 24, 31 (2016)

[18] X. Li, X. Li, J. Zhu, X. Ye, G. Tang, Scr. Mater. 112, 23-27 (2016)

[19] P. Song, X. Li, W. Ding, J. Chen, Acta Metall. Sin. (Engl. Lett.) 4, 27 (2014)

[20] C. Li, S. Jiang, K. Zhang, Z. Zhao, Acta Metall. Sin. (Engl. Lett.) 2, 25 (2012)

[21] Y. Sun, H. Ahlatci, Mater. Des. 5, 32 (2011)

[22] X. Li, X. Wang, M. Saunders, A. Suvorova, L. Zhang, Y. Liu, M. Fang, Z. Huang, T.B. Sercombe, Acta Mater. 15, 95 (2015)

[23] X. Wu, N. Tao, Y. Hong, B. Xu, J. Lu, K. Lu, Acta Mater. 8, 50 (2002)

[24] X. Tong, H. Zhang, D. Li, Sci. Rep. 5 (2015)

[25] Y. Liu, B. Jin, J. Lu, Mater. Sci. Eng. A 11, 636 (2015)

[26] X. Huang, N. Hansen, N. Tsuji, Science 5771, 312 (2006)

[27] B. Malard, J. Pilch, P. Sittner, R. Delville, C. Curfs, Acta Mater. 4, 59 (2011)

[28] X. Li, G. Tang, J. Kuang, X. Li, J. Zhu, Mater. Sci. Eng. A 26, $612(2014)$

[29] X. Li, X. Li, S.Z. Kure-Chu, G. Tang, Metall. Mater. Trans. A 2 , 49 (2018)

[30] W. Li, Y. Shen, H. Liu, Y. Wang, W. Zhu, C. Xie, J. Mater. Res. 9, 31 (2016)

[31] T.J. Rupert, J.R. Trelewicz, C.A. Schuh, J. Mater. Res. 09, 27 (2012)

[32] J. Hu, Y. Shi, X. Sauvage, G. Sha, K. Lu, Science 6331, 355 (2017)

[33] G. Tang, J. Zhang, M. Zheng, J. Zhang, W. Fang, Q. Li, Mater. Sci. Eng. A 1, 281 (2000)

[34] Y. Zhu, S. To, W. Lee, X. Liu, Y. Jiang, G. Tang, Mater. Sci. Eng. A 1, 501 (2009)

[35] R. Qin, Sci. Rep. 7 (2017) 